\title{
A influência da dor na qualidade de vida de idosos portadores de Diabetes Mellitus
}

\author{
The influence of pain on elderly diabetics' quality of life \\ La influencia del dolor en la calidad de vida de ancianos portadores de Diabetes Mellitus
}

\author{
Fernanda Thais Ferreira de Paiva', Luciano Ramos de Lima", Mani Indiana Funez"I', \\ Cris Renata Grou Volpe ${ }^{\prime V}$, Silvana Schwerz Funguetto ${ }^{V}$, Marina Morato Stival ${ }^{V I}$
}

\begin{abstract}
RESUMO
Objetivo: avaliar a dor e suas repercussões na qualidade de vida de idosos com Diabetes Mellitus. Método: estudo transversal com 196 idosos com Diabetes Mellitus, em uma unidade básica de saúde do Distrito Federal. Utilizados os questionários de qualidade de vida WHOQOL-OLD e WHOQOL-BREF. Resultados: dos 196 idosos, 54,6\% eram do sexo masculino, com média de idade de $67,4( \pm 6,5)$ anos, com predomínio entre 60 e 70 anos $(72,9 \%)$. Por meio das pontuações do WHOQOL-BREF e WHOQOLOLD, observou-se que aqueles que não relataram dor apresentaram melhores pontuações de qualidade de vida, exceto no domínio físico ( $M=53,94$ e $M=59,26)$ e na faceta autonomia $(M=56,68$ e $M=58,19) p \leq 0,05$. Conclusão: o estudo demonstrou uma influência negativa da dor na qualidade de vida de idosos com Diabetes Mellitus, sendo necessário que os enfermeiros estejam atentos, pois a mesma está intrinsicamente ligada à qualidade do tratamento.
\end{abstract}

Descritores: Idosos; enfermagem; atenção primária à saúde; qualidade de vida.

\section{ABSTRACT}

Objective: to evaluate pain and its repercussions on the quality of life of elderly people with Diabetes Mellitus. Methods: in this cross-sectional study of 196 older adults with Diabetes Mellitus, WHOQOL-OLD and WHOQOL-BREF quality of life questionnaires were applied at a primary health care facility in the Federal District. Results: of the 196 participants, 54.6\% were males, mean age $67.4( \pm 6.5)$ years, predominantly 60 to 70 years $(72.9 \%)$. The WHOQOL-BREF and WHOQOL-OLD scores showed that those who did not report pain returned higher quality of life scores, except in the physical domain $(M=53.94$ and $M=59.26)$ and in the autonomy facet $(M=56.68$ and $M=58.19 ; p \leq 0.05)$. Conclusion: the study showed the adverse influence of pain on quality of life of older adults with Diabetes Mellitus. Nurses need to be attentive to this, because it is intrinsically connected with treatment quality.

Descriptors: Aged; nursing; primary health care; quality of life.

\section{RESUMEN}

Objetivo: evaluar el dolor y sus repercusiones en la calidad de vida de ancianos con Diabetes Mellitus. Método: estudio transversal junto a 196 ancianos con Diabetes Mellitus, en una unidad básica de salud del Distrito Federal. Se utilizaron los cuestionarios de calidad de vida WHOQOL-OLD y WHOQOL-BREF. Resultados: de los 196 ancianos, un 54,6\% era del sexo masculino, con promedio de edad de $67,4( \pm 6,5)$ años, con predominio entre 60 y 70 años $(72,9 \%)$. Mediante las puntuaciones del WHOQOL-BREF y WHOQOL-OLD, se observó que aquéllos que no reportaron dolor presentaron mejores puntuaciones de calidad de vida, excepto en el dominio físico $(M=53,94$ y $M=59,26)$ y en lo que respecta la autonomía $(M=56,68$ y $M=58,19)$ $\mathrm{p} \leq 0,05$. Conclusión: el estudio demostró una influencia negativa del dolor en la calidad de vida de ancianos con Diabetes Mellitus. Siendo así, es necesario que los enfermeros estén atentos, pues la calidad de vida está intrínsecamente relacionada a la del tratamiento.

Descriptores: Anciano; enfermería; atención primaria de salud; calidad de vida.

\section{INTRODUÇÃO}

Segundo pesquisas realizadas em 2011 pelo Instituto Brasileiro de Geografia e Estatística (IBGE), o número de idosos no Brasil dobrou nos últimos 20 anos, chegando a 23,5 milhões'. $O$ avanço da idade pode levar ao aparecimento de doenças e agravos crônicos, que requerem acompanhamento e tratamento contínuo. Essas doenças tendem a se manifestar de forma expressiva nos idosos, e geralmente estão associadas a outras patologias².

\footnotetext{
'Bolsista de Inciação científica da Universidade de Brasília - Faculdade de Ceilândia, Brasília, DF, Brasil. E-mail: fernandinhathays@gmail.com "Doutorando PPCTS/UNB. Professor Assistente do curso de Enfermagem da Universidade de Brasília/Campus Ceilândia, DF, Brasil. E-mail: ramosll@unb.br I'Doutora. Professora Adjunta do curso de Enfermagem da Universidade de Brasília/Campus Ceilândia, DF, Brasil. E-mail: mani@unb.br IV Doutora. Professora Adjunta do curso de Enfermagem da Universidade de Brasília/Campus Ceilândia, DF, Brasil. E-mail: crgrou@unb.br vDoutora. Professora Adjunta do curso de Enfermagem da Universidade de Brasília/Campus Ceilândia, DF, Brasil. E-mail: silvanasf@unb.br viDoutora. Professora Adjunta do curso de Enfermagem da Universidade de Brasília/Campus Ceilândia, DF, Brasil. E-mail: marinamorato@unb.br VIIAgradecimento à Fundação Universidade de Brasília que financiou esta pesquisa. Processo: 10.1257 / agosto de 2016.
} 
Com o evidente o aumento no número de idosos, observa-se também uma maior prevalência de Doenças Crônicas Não Transmissíveis (DCNT), que são referidas como uma das principais causas de morte em todo mundo ${ }^{3}$. Segundo a Organização Mundial da Saúde (OMS), de todos os óbitos ocorridos no ano de 2008 em todo o mundo, 63\% foram devido às DCNT e/ou suas complicações. No Brasil, esse número foi um pouco maior, chegando à $74 \%$ das mortes em $2012^{4}$. Entre as DCNT mais prevalentes em idosos, observa-se o Diabetes Mellitus (DM) ${ }^{5}$.

Segundo a International Diabetes Federation (IDF), é estimado que a população mundial de diabéticos esteja em 415 milhões de pessoas, devendo atingir 642 milhões até 2040. Além disso, 75\% das pessoas com DM estão nos países de baixa e média renda. Nos países da América Central e do Sul, a prevalência de DM foi estimada em 29,6 milhões de pessoas e projetada para 48,8 milhões, em 2040 . No ano de 2015 , o Brasil foi o quarto país com mais adultos com DM no mundo, com uma população de diabéticos estimada em 14,3 milhões, ficando atrás apenas da China (109,6 milhões), Índia (69,2 milhões) e Estados Unidos da América (29,3 milhões) ${ }^{5}$.

O aumento no número de casos da DCNT está associado a vários fatores, como o envelhecimento da população, hábitos de vida não saudáveis, dieta inadequada e obesidade. O DM mal controlado gera diversas complicações que comprometem a produtividade, a qualidade de vida (QV) e a expectativa de vida do indivíduo ${ }^{6}$. O DM, que por ser uma condição crônica, necessita de um controle maior e tratamento contínuo, quando aliado a complicações, como a dor, constitui um fator importante na determinação da QV7.

A QV é um aspecto importante no DM, pois sua redução leva a uma diminuição do autocuidado, o que, por sua vez, ocasiona um pior controle glicêmico, aumento dos riscos de complicações, tanto a curto quanto a longo prazo ${ }^{8}$. Muitos fatores podem influenciar e afetar a QV dos diabéticos, como a idade, a situação financeira, a escolaridade, a prática de atividade física, a nutrição, as comorbidades associadas, os hábitos de vida, o tipo de tratamento adotado e as complicações, pois afetam o estado físico, funcional e psicológico, e de forma geral, o bem-estar do indivíduo ${ }^{2,9}$.

Entre estes fatores destaca-se a presença da dor, principalmente crônica, como uma complicação do DM que influencia diretamente na vida do idoso, especialmente na sua QV. A dor do diabético tem sido descrita como intensa, que causa desconforto e/ou insensibilidade no pés e/ou panturrilhas, em forma de picada, choque, queimação, dormência e câimbras, e ainda, um prejuízo que incomoda e faz com que os pacientes despertem a noite devido ao desconforto da dor, principalmente quando relacionada à neuropatia diabética ${ }^{10}$.

Resultados de pesquisas reconhecem uma pior QV em idosos com DM, quando investigada com o instrumento WHOQOL-BREF ${ }^{6,8,11}$. Vale ressaltar estudo realizado com diabéticos na atenção primária que evidenciou uma correlação negativa entre a intensidade de dor e índices de QV, ou seja, quanto maior intensidade de dor do diabético pior era sua $\mathrm{QV}^{12}$. Sabe-se que as abordagens da maioria dos estudos concentram-se na identificação dos principais domínios afetados na QV do idoso. Observa-se, portanto, que são escassas pesquisas que relacionem a investigação da QV com a mensuração da dor.

Diante do exposto e considerando as especificidades do idoso, torna-se necessário o desenvolvimento de estudos que investiguem a relação entre dor e QV na população de idosos diabéticos, uma vez que se observa um crescente aumento das DCNT. Conhecer essa relação pode permitir ao enfermeiro promover uma melhor QV aos idosos que convivem com a dor e a presença de uma doença crônica progressiva, o DM, por meio de um cuidado planejado baseado em resultados de investigações realizadas com instrumentos validados e de fácil aplicação. Sendo assim, o objetivo deste estudo foi avaliar a dor e suas repercussões na qualidade de vida de idosos com Diabetes Mellitus.

\section{MetOdOLOGiA}

Trata-se de um estudo descritivo, transversal de abordagem quantitativa. A pesquisa ocorreu em uma Unidade Básica de Saúde (UBS) do Distrito Federal, em 2016. A população do estudo foi composta por 500 idosos com Diabetes Mellitus cadastrados no Grupo de Diabéticos da UBS. A amostra por conveniência foi constituída de acordo com o seguinte critério de inclusão: idosos com 60 anos ou mais que faziam parte do grupo de diabéticos da UBS. O idoso foi abordado no momento da reunião do Grupo e convidado a participar da pesquisa. A coleta dos dados ocorreu entre setembro e novembro de 2016. Ao final, 196 idosos constituíram a amostra final do estudo.

A entrevista ocorreu em local reservado, após a assinatura do TCLE, e foram utilizados três instrumentos. O primeiro foi um questionário estruturado, elaborado pelos pesquisadores e previamente testado, composto por perguntas direcionadas ao perfil socioeconômico e demográfico (sexo, idade, estado civil, escolaridade, renda e ocupação), e avaliação da dor. A intensidade de dor foi mensurada por meio da Escala Numérica Visual (ENV) que varia de 0 a 10 pontos, na qual 0 (zero) significa sem dor e 10 (dez) a pior dor possível. A escala numérica foi categorizada de acordo com a intensidade de dor, sendo de 1-3 pontos (dor leve), 4-6 (dor moderada), 7-9 (dor intensa) e 10 (pior dor possível) ${ }^{13}$. 
O segundo instrumento foi o WHOQOL-BREF, que consiste em uma versão resumida do questionário proposto pela OMS para avaliar a QV, sendo dividido em quatro domínios: Físico, Psicológico, Relações sociais e Meio ambiente. Por fim o terceiro questionário utilizado foi o WHOQOL-OLD, ferramenta da OMS para avaliar a QV de idosos, composto por seis facetas: Sensorial; Habilidades; Autonomia; Atividades passadas, presentes e futuras; Participação social; Morte e morrer; e Intimidade. Ambos os instrumentos de QV foram validados no Brasil ${ }^{14,15}$. Para cada domínio e faceta foi calculado um escore, que variava de 0 a 100, sendo que quanto maior o escore, melhor a QV.

Para análise de dados, a primeiro momento foi criado um banco de dados no software Package for the Social Sciences (SPSS ${ }^{\circledR}$ ) versão 21.0. Posteriormente, de acordo com as instruções do instrumento, foram calculados os escores totais de cada respondente, bem como os valores médios por grupo de cada um dos fatores e domínios, sendo assim realizada uma análise quantitativa descritiva, onde foram calculadas frequências relativas, absolutas, médias e desvio padrão dos resultados obtidos. No último momento, para analisar a normalidade das variáveis utilizou-se o teste Komogorov-Smirnov. O teste $t$ de Student foi utilizado para analisar as diferenças entre as médias das medidas de QV. Para verificar diferenças entre proporções foi utilizado o teste qui-quadrado. Os valores de $p<0,05$ foram considerados estatisticamente significantes.

A pesquisa foi aprovada pelo Comitê de Ética em Pesquisa da Secretaria do Estado de Saúde do Distrito Federal (CAAE: 14557613.1.0000.5553), e os sujeitos foram esclarecidos pelos pesquisadores sobre princípios éticos resolução CNS 466/2012.

\section{RESULTADOS}

Os dados referentes às características sociodemográficas e de aferição da dor são demonstrados na Tabela 1.

Tabela 1: Distribuição das variáveis demográficas e socioeconômicas dos idosos com diabetes mellitus de acordo com a intensidade de

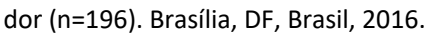

\begin{tabular}{|c|c|c|c|c|c|c|c|c|c|}
\hline \multirow[t]{2}{*}{ Variáveis } & \multicolumn{2}{|c|}{$\begin{array}{c}\text { Dor } \\
\text { Leve/Moderada } \\
\mathbf{n}=\mathbf{7 1}\end{array}$} & \multicolumn{2}{|c|}{$\begin{array}{c}\text { Dor } \\
\text { Intensa } \\
\mathbf{n}=96\end{array}$} & \multicolumn{2}{|c|}{$\begin{array}{c}\text { Sem } \\
\text { dor } \\
\mathbf{n}=\mathbf{2 9}\end{array}$} & \multicolumn{2}{|c|}{ Total } & \multirow[t]{2}{*}{$\mathbf{p}$} \\
\hline & $n$ & $\%$ & $\mathbf{n}$ & $\%$ & $\mathbf{n}$ & $\%$ & $\mathbf{n}$ & $\%$ & \\
\hline Sexo & & & & & & & & & 0,290 \\
\hline Feminino & 27 & 38,0 & 48 & 50,0 & 14 & 48,3 & 89 & 45,4 & \\
\hline Masculino & 44 & 62,0 & 48 & 50,0 & 15 & 51,7 & 107 & 54,6 & \\
\hline Idade (anos) & & & & & & & & & 0,924 \\
\hline $60-70$ & 52 & 73,2 & 70 & 72,9 & 21 & 72,4 & 143 & 73,0 & \\
\hline $71-80$ & 14 & 19,7 & 22 & 22,9 & 6 & 20,7 & 42 & 21,4 & \\
\hline$>80$ & 5 & 7,1 & 4 & 4,2 & 2 & 6,9 & 11 & 5,6 & \\
\hline Estado civil & & & & & & & & & 0,008 \\
\hline Solteiro & 31 & 43,7 & 35 & 36,5 & 14 & 48,3 & 80 & 40,8 & \\
\hline Casado & 21 & 29,6 & 25 & 26,0 & 12 & 41,4 & 58 & 29,5 & \\
\hline Viúvo & 10 & 14,1 & 13 & 13,5 & 1 & 3,4 & 24 & 12,4 & \\
\hline Divorciado & 9 & 12,7 & 23 & 23,9 & 2 & 6,9 & 34 & 17,3 & \\
\hline Escolaridade & & & & & & & & & 0,052 \\
\hline Analfabeto & 15 & 21,1 & 19 & 19,8 & 4 & 13,8 & 38 & 19,4 & \\
\hline Ensino & 50 & 70,4 & 74 & 77,1 & 20 & 68,9 & 144 & 73,5 & \\
\hline \multicolumn{10}{|l|}{ Fundamental } \\
\hline Ensino Médio & 5 & 7 & 3 & 3,1 & 5 & 15,2 & 13 & 6,6 & \\
\hline Superior & 1 & 1,4 & 0 & 0 & 0 & 0 & 1 & 0,5 & \\
\hline Renda Mensal & & & & & & & & & 0,046 \\
\hline Até 1 SM & 41 & 57,7 & 65 & 67,7 & 14 & 48,3 & 120 & 61,22 & \\
\hline 1 a 3 SM & 22 & 31,0 & 24 & 25,0 & 11 & 37,9 & 57 & 29,1 & \\
\hline$>4 \mathrm{SM}$ & 8 & 11,3 & 7 & 7,3 & 4 & 13,9 & 19 & 9,7 & \\
\hline Ocupação & & & & & & & & & 0,012 \\
\hline Desempregado & 20 & 28,2 & 14 & 14,6 & 7 & 24,1 & 41 & 20,9 & \\
\hline Aposentado & 45 & 63,4 & 60 & 62,5 & 19 & 65,5 & 124 & 63,3 & \\
\hline Outros & 6 & 8,5 & 22 & 22,9 & 3 & 10,3 & 31 & 15,8 & \\
\hline
\end{tabular}


Entre os 196 idosos participantes do estudo, 54,6\% eram do sexo masculino, com idade média de $67,47 \pm 6,59$ anos, $73,0 \%$ tinham entre 60 e 70 anos, $40,8 \%$ eram solteiros, $73,5 \%$ tinham o ensino fundamental, $63,3 \%$ eram aposentados e $61,2 \%$ tinham uma renda mensal de até um salário mínimo.

Referente à análise da dor, percebeu-se que 49\% relataram dor intensa, 36\% dor leve/moderada e $15 \%$ não referiram dor. Em relação às variáveis demográficas e socioeconômicas foram observadas diferenças significativas no estado civil solteiro, na baixa renda mensal e na aposentadoria dos idosos. Idosos diabéticos solteiros apresentaram maior prevalência de dor intensa $(36,5 \%)$ quando comparados aos casados $(26,0 \%)(p=0,008)$. Quanto à renda mensal, verificou-se que aqueles idosos diabéticos com renda de até um salário mínimo referiram mais dor intensa (67,7\%) do que aqueles com maior renda $(25,0 \%)(p=0,046)$. Por fim, um maior número de idosos aposentados queixou dor intensa $(62,5 \%)$ quando comparados aos idosos que estavam desempregados no momento da pesquisa $(14,6 \%)(p=0,012)$.

Os escores de QV avaliada por meio dos questionários WHOQOL-BREF e WHOQOL-OLD são apresentados na Tabela 2, de acordo com a presença ou não da dor.

Tabela 2: Médias de escores obtidas pelo WHOQOL-BREF e WHOQOL-OLD e relato de dor entre os idosos diabéticos ( $n=196)$. Brasília, DF, Brasil, 2016.

\begin{tabular}{|c|c|c|c|c|c|}
\hline & \multicolumn{2}{|c|}{ Dor não referida } & \multicolumn{2}{|c|}{ Dor referida } & \multirow[t]{2}{*}{$\mathbf{p}$} \\
\hline & $\mathbf{M}$ & DP & $\mathbf{M}$ & DP & \\
\hline \multicolumn{6}{|l|}{ WHOQOL-BREAF } \\
\hline Físico & 53,94 & 14,76 & 59,26 & 14,18 & 0.053 \\
\hline Psicológico & 64,36 & 14,79 & 59,45 & 14,83 & 0.656 \\
\hline Relações sociais & 67,13 & 19,66 & 65,79 & 21,91 & 0.091 \\
\hline Meio ambiente & 60,56 & 17,78 & 55,08 & 15,31 & 0.046 \\
\hline \multicolumn{6}{|l|}{ WHOQOL-OLD } \\
\hline Funcionamento sensorial & 71,33 & 16,83 & 62,23 & 24,45 & 0.048 \\
\hline Autonomia & 56,68 & 14,45 & 58,19 & 18,67 & 0.879 \\
\hline Atividades passadas, presentes e futuras & 66,81 & 20,19 & 65,98 & 19,11 & 0.011 \\
\hline Participação social & 61,85 & 20,95 & 59,84 & 19,01 & 0.026 \\
\hline Morte e morrer & 58,18 & 31,03 & 55,12 & 32,20 & 0.354 \\
\hline Intimidade & 74,35 & 24,51 & 68,67 & 23,27 & 0.522 \\
\hline
\end{tabular}

Observa-se que os idosos que não referiram dor apresentaram melhores escores de QV do que aqueles que queixaram dor, exceto nos domínios Físico e na faceta Autonomia. No grupo de idosos com DM que não referiram dor uma melhor QV foi observada no domínio Relações sociais $(67,13 \pm 19,66)$ e faceta Intimidade $(74,35 \pm 24,51)$ e uma pior QV no domínio Físico $(53,94 \pm 14,76)$ e faceta Autonomia $(56,68 \pm 14,45)$.

Por outro lado, no grupo de idosos com DM que queixaram dor uma melhor QV foi observada no domínio Relações sociais $(65,79 \pm 21,91)$ e faceta Intimidade $(68,67 \pm 23,27)$, e uma pior QV no domínio Meio ambiente $(55,08 \pm 15,31)$ e faceta Morte e morrer $(55,12 \pm 32,20)$.

Na Tabela 3 é demonstrada a comparação entre médias dos escores de QV entre o grupo que referiu dor leve/moderada e o grupo que referiu dor intensa.

Verificou-se que aqueles idosos diabéticos que queixaram dor intensa apresentaram escores menores de QV do que aqueles com dor leve/moderada, exceto no domínio Físico ( $p=0.053)$. Diferenças significativas foram observadas no domínio Meio ambiente $(\mathrm{p}=0.046)$ indicando que idosos com dor intensa apresentam uma pior QV nesse domínio.

$\mathrm{Na}$ análise dos resultados da QV avaliada pelo WHOQOL-OLD, o grupo de idosos com dor intensa apresentou pior QV nas facetas Funcionamento sensorial $(p=0,048)$, Atividades passadas, presentes e futuras $(p=0,011)$ e Participação social $(p=0,026)$ (Tabela 3$)$. 
Tabela 3: Comparação entre médias dos escores dos questionários WHOQOL-BREF e WHOQOL-OLD e a intensidade da dor em idosos com diabetes. Brasília, DF. Brasil, 2016.

\begin{tabular}{|c|c|c|c|c|c|}
\hline & \multicolumn{5}{|c|}{ Intensidade da dor } \\
\hline & \multicolumn{2}{|c|}{ Leve/Moderada } & \multicolumn{2}{|c|}{ Intensa } & \multirow[b]{2}{*}{$\mathbf{p}$} \\
\hline & $\mathbf{M}$ & DP & $\mathbf{M}$ & DP & \\
\hline \multicolumn{6}{|l|}{ WHOQOL-BREF } \\
\hline Físico & 59.05 & 12.67 & 59.41 & 15.27 & 0.053 \\
\hline Psicológico & 61.03 & 14.94 & 58.28 & 14.72 & 0.656 \\
\hline Relações Sociais & 67.13 & 19.66 & 65.79 & 21.91 & 0.091 \\
\hline Meio Ambiente & 57.83 & 15.19 & 53.05 & 15.16 & 0.046 \\
\hline \multicolumn{6}{|l|}{ WHOQOL-OLD } \\
\hline Funcionamento sensorial & 66.1 & 23.94 & 29.37 & 24.55 & 0.048 \\
\hline Autonomia & 60.47 & 18.26 & 56.51 & 18.89 & 0.879 \\
\hline Atividades passadas, presentes e futuras & 70.33 & 17.22 & 62.76 & 18.88 & 0.011 \\
\hline Participação Social & 63.73 & 15.9 & 56.96 & 20.63 & 0.026 \\
\hline Morte e morrer & 55.45 & 33.17 & 54.88 & 31.63 & 0.354 \\
\hline Intimidade & 70.07 & 23.83 & 67.64 & 22.92 & 0.522 \\
\hline
\end{tabular}

\section{DISCUSSÃO}

Neste estudo foi observado que a maioria dos idosos com DM referiu dor intensa, que foi relacionada ao estado civil solteiro, à baixa renda mensal e à aposentadoria dos idosos. Esses resultados corroboram com aqueles evidenciados em outro estudo realizado com diabéticos no Pará ${ }^{16}$. Tais resultados denotam uma maior preocupação, já que a presença de um companheiro poderia favorecer os idosos com doenças crônicas, atuando como um suporte e encorajando o autocuidado. Portanto, esse dado reflete a necessidade maior de uma atenção e maior apoio por parte da equipe de saúde, visando criar um vínculo com esses idosos, e contribuindo para uma melhor adesão ao plano terapêutico ${ }^{17}$.

Além disso, a baixa renda é um fator que pode comprometer o estado de saúde e a QV dos idosos, dificultando a adesão ao tratamento, e principalmente a terapêutica não medicamentosa, que inclui a mudança dos hábitos alimentares e prática de exercício físico, fundamental para um controle glicêmico ideal para evitar complicações do $\mathrm{DM}^{18}$.

O presente estudo demonstrou que o grupo que referiu dor apresentou escores menores de QV do que o grupo que não referiu dor, ou seja, apresentaram uma menor QV. Cabe ressaltar um estudo realizado com idosos em Montes Claros (MG) que observou um impacto negativo da dor na QV dos pacientes diabéticos, principalmente naqueles com mais de 10 anos de progressão da doença, uma vez que um maior tempo de evolução do DM pode refletir em uma maior intensidade de dor e desconfortos causados por ela, que consequentemente ocasiona em uma maior interferência nas atividades cotidianas ${ }^{19}$.

É válido ressaltar que a cronificação da dor está relacionada à neuropatia diabética (ND), uma das complicações do DM. A prevalência de neuropatia diabética dolorosa tem aumentado, pois um a cada cinco pacientes diabéticos têm dor crônica com característica de dor neuropática ${ }^{20}$. Estudos têm demonstrado o impacto negativo desta complicação na QV dos pacientes. Neste contexto, em uma pesquisa foi investigada a relação entre neuropatia diabética dolorosa e QV em pacientes com ND. Participaram 154 pacientes e observaram que a QV foi menor em pacientes com neuropatia dolorosa ${ }^{21}$.

Neste estudo aqueles idosos com DM que queixaram dor obtiveram menores escores de QV no domínio Meio Ambiente e na faceta Morte e Morrer. Resultados contrários foram observados no estudo com idosos em Uberaba (MG), onde a faceta Morte e Morrer obteve maior pontuação ${ }^{22}$. Para os idosos, a própria idade aliada com complicações de saúde pode trazer a ideia de um futuro mais curto e uma aproximação com a ideia da morte, podendo causar uma reação de medo e não aceitação ${ }^{23}$.

Os domínios e facetas com menores escores de QV que foram significativamente associados à presença de dor foram Meio ambiente, Funcionamento sensorial, Atividades passadas, presentes e futuras e Participação social. A dor apresenta-se como um fator limitante para realização das atividades cotidianas e tarefas de vida diária, podendo influenciar negativamente no convívio social e na $\mathrm{QV}^{24}$. Uma pesquisa associou a influência da dor crônica de 50 idosos à QV por meio de utilização do instrumento WHOQOL-BREF. Os resultados evidenciaram que houve uma influência negativa da dor crônica na QV dos idosos, principalmente, no domínio físico e nas relações sociais ${ }^{11}$.

A faceta Funcionamento sensorial avalia o impacto da perda das habilidades sensoriais na qualidade vida dos idosos. Os órgãos sensoriais permitem o relacionamento do idoso com o meio em que vive. É sabido que a diminuição 
da sensibilidade comumente afeta os pacientes com DM, principalmente em relação aos pés. Em igual teor, o DM em progressão tende a ocasionar complicações microvasculares como a retinopatia que quando associadas às modificações relacionadas ao envelhecimento, como a diminuição da capacidade auditiva, afeta os órgãos sensoriais do idoso. Adicionalmente, a presença da dor pode potencializar o prejuízo na percepção sensorial relacionada aos processos inflamatórios persistentes da hiperglicemia, relacionados ao descontrole do $\mathrm{DM}^{25}$.

Um estudo realizado com 146 mulheres idosas com DM também evidenciou uma pior QV no domínio Meio ambiente $(M=38,91)$, demonstrando a importância do incentivo à responsabilização pessoal da sociedade de criar ambientes amistosos para idosos, estimulando assim, a solidariedade entre as gerações ${ }^{26}$. Cabe ressaltar que esse domínio avalia a segurança física, proteção, o ambiente no lar, os recursos financeiros, as oportunidades de adquirir novas informações e habilidades, a poluição/ruído/trânsito/clima e o transporte ${ }^{14}$.

Na comparação entre os idosos que sentiam dor, de acordo com a intensidade dessa dor, evidenciou-se que os idosos que referiram dor intensa apresentaram pior QV no domínio Meio ambiente do que os idosos com dor leve/moderada. Assim como em um estudo realizado em João Pessoa (PB) o maior impacto da QV foi no domínio relacionado ao ambiente ${ }^{27}$, o que pode estar relacionado com a infraestrutura da região, com o índice de criminalidade e com as dificuldades de acesso aos serviços de saúde, principalmente, por ser uma região de baixo nível socioeconômico ${ }^{28}$. Esse resultado indica que as equipes de saúde devem buscar estratégias que melhorem as condições de saúde dos idosos que vivem em áreas com acesso limitado aos serviços de saúde, e poucas oportunidades de lazer ${ }^{29}$. A manutenção e promoção de segurança é fundamental para o desenvolvimento de atividades de lazer, já que muitas vezes estes idosos convivem com a presença de dor crônica que limita a realização dessas atividades.

Os idosos com DM que referiram dor intensa também apresentaram pior QV nas facetas Funcionamento sensorial, Atividades passadas, presentes e futuras e Participação social. Os piores escores de QV na faceta Funcionamento sensorial nos indivíduos que sentem dor intensa também foi encontrada no estudo realizado na cidade de Uberaba (MG) com idosos diabéticos ${ }^{29}$. A respeito disso, sabe-se que à medida que as pessoas envelhecem, o corpo sofre mudanças estruturais e funcionais no sistema sensorial, além desse processo natural, complicações do DM, como a dor, podem acentuar essas mudanças, causando um impacto maior na QV ${ }^{30}$.

Esse resultado indica a necessidade de refletir sobre a possibilidade de um maior risco de acidentes relacionados a alterações sensoriais e sobre a ideia de que esses idosos podem se tornar menos ativos devido às suas limitações. Outros pesquisadores reforçam ainda, que quanto menos ativo o idoso, menor a sua satisfação com a vida e, consequentemente, pior a sua $\mathrm{QV}^{31}$.

Nesse sentido, um estudo analisou o efeito das quedas e suas consequências na QV de idosos de uma comunidade de baixa renda do município do Rio de Janeiro. A análise demonstrou que há influência das quedas na QV dos idosos estudados, pois em todos os domínios do WHOQOL-BREF houve redução nas médias do grupo que caiu no último ano em relação aos que não caíram ${ }^{32}$. Enfatiza-se que o processo de envelhecimento cursa com alterações do sistema musculoesquelético que muitas vezes está sobrecarregado de alterações como artrite, artrose que tem a presença de dor em seu cotidiano, que limita e traz instabilidade de marcha que pode contribuir com as quedas.

A faceta Atividades passadas, presentes e futuras indica a satisfação dos idosos quanto às conquistas ocorridas no decorrer da vida e acerca das esperanças futuras, coisas que ainda se anseiam. Sendo assim, é de suma importância que a equipe de enfermagem promova ações em saúde que ajude o idoso na busca de atividades que lhe tragam realizações pessoais e uma melhora nas perspectivas para o futuro ${ }^{33}$. Sabe-se que as expectativas do indivíduo podem sofrer influência de fatores culturais, socioeconômicos, nível de dependência, acesso aos serviços de saúde e além disso, as comorbidades podem levar o indivíduo a diminuir suas perspectivas em relação ao seu futuro.

Em relação à faceta Participação social resultado semelhante foi observado em um estudo com 68 idosos diabéticos na Paraíba. Os autores evidenciaram uma pior QV nos aspectos sociais demonstrando o comprometimento da participação dos idosos em grupos sociais em virtude das dificuldades que o DM impõe ${ }^{34}$.

Os idosos com dor intensa apresentaram um maior escore na faceta Intimidade, assim como na pesquisa realizada

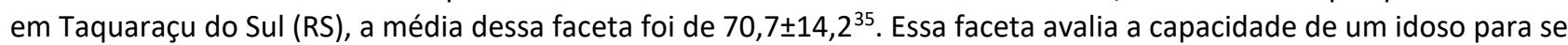
relacionar com outras pessoas, sendo uma área que deve ser constantemente observada pelos enfermeiros, pois a rede de relacionamentos dos idosos são extremamente importantes para a forma que essas pessoas vão lidar com a doença ${ }^{29}$.

A pontuação mais alta no domínio das relações sociais para ambos os grupos é semelhante à encontrada com idosos com DM na Nigéria ${ }^{36}$, e em outro estudo realizado também com idosos diabéticos em São Paulo ${ }^{37}$. Esse domínio avalia as relações pessoais, o suporte social e a atividade sexual. Esse resultado pode ser explicado pelas atividades físicas oferecidas aos participantes todos os dias na UBS. Tais atividades configuram-se como oportunidade de socialização dos idosos. Outro estudo reforça o valor da atividade física regular para melhorar o aspecto físico e 
funcional dos idosos, mas que não se deve esquecer da importância que essas atividades têm nos aspectos sociais, devendo ser incluídas atividades para promoção e manutenção das habilidades sociais dessa população ${ }^{38}$.

Isto posto, é fundamental o papel do enfermeiro na investigação e controle da dor de idosos diabéticos como uma das estratégias de enfrentamento dos idosos no manejo do DM. Sabe-se que o DM é um fator que contribui para a presença de outras comorbidades como a depressão, sendo a dor somada a este fator, que pode ainda potencializar as complicações do $\mathrm{DM}^{12}$.

A equipe de saúde, especialmente o enfermeiro, deve atentar-se para estes fatores que interferem no cotidiano e na QV dos diabéticos, para propor ações a serem incorporadas às intervenções de enfermagem nesta população com vistas a potencializar a QV. Adicionalmente, o enfermeiro deve reconhecer de maneira precoce o surgimento da dor, como um sinal da neuropatia diabética, a fim de prevenir futuras complicações como amputações.

\section{CONCLUSÃO}

A dor interferiu negativamente na qualidade de vida de idosos com Diabetes Mellitus principalmente, no domínio Físico e faceta Autonomia.

Os resultados deste estudo comprovam a importância de se avaliar a influência da dor na QV de idosos diabéticos, proporcionando uma análise do impacto negativo dessa complicação do DM nas questões físicas e psicossociais desses pacientes. Além disso, fica evidente a necessidade que as equipes de saúde, especialmente os enfermeiros, estejam atentos à todas variáveis que possam interferir na QV desses idosos, visto que a mesma está intrinsicamente ligada a adesão ao plano terapêutico proposto, bem como a qualidade desse tratamento. Portanto, estudos como este possibilitam o direcionamento do planejamento de ações que atendam às necessidades específicas dessa população.

\section{REFERÊNCIAS}

1. Instituto Brasileiro de Geografia e Estatística - IBGE. Sinopse do Senso Demográfico de 2010[Internet]; Rio de Janeiro: IBGE; 2011. [cited 2018 Dec 15] 261p. Available from: https://biblioteca.ibge.gov.br/visualizacao/livros/liv49230.pdf

2. Sousa MC, Dias FA, Nascimento JS, Tavares DMS. Correlation of quality of life with knowledge and attitude of diabetic elderly. Invest. educ. Enferm [Internet]. 2016 [cited 2018 Dec 15]; 34(1):180-8. DOI: http://dx.doi.org/10.17533/udea.iee.v34n1a20

3. Rocha-Brischiliari SC, Dell Agnolo CM, Gravea ÂAF, Lopes TCR, Carvalho MD de B, Pelloso SM. Doenças crônicas não transmissíveis e associação com fatores de risco. Rev bras cardiol [Internet]. 2014 [cited 2018 Dec 15]; 27(1):531-8. Available from: http://www.rbconline.org.br/wpcontent/uploads/Art_52_RBC_27_1_Catia_Agnollo_Artigo_Original1.pdf

4. World Health Organization. Mortality and burden of disease. Noncommunicable Diseases (NCD) Country Profiles, 2014 [Internet]; Geneva: WHO; 2014 [cited 2018 Dec 15]; 210p. Available from: http://www.who.int/nmh/countries/en/

5. International Diabetes Federation. IDF Diabetes Atlas - Seventh Edition, 2015. [Internet]; Brussels, Belgium: International Diabetes Federation; 2015 [cited 2018 Dec 15]; 140p. Available from: http://www.diabetesatlas.org

6. Paskulin L, Vianna L, Molzhan A.E. Factors associated with quality of life of Brazilian older adults. Int. Nur. Rev. [Internet]. 2009 [cited 2018 Dec 15]; 56(1):109-15. Available from: DOI: https://doi.org/10.1111/j.1466-7657.2008.00671.x

7. Silva LD, Pazos AL. A influência da dor na qualidade de vida do paciente com lesão crônica de pele. Rev. Enferm. UERJ. [Internet]. 2005 [cited 2018 Dec 15]; 13(3):375-81. Available from: http://www.facenf.uerj.br/v13n3/v13n3a13.pdf

8. Jain V, Shivkumar S, Gupta O. Health-Related Quality of Life (Hr-Qol) in Patients with Type 2 Diabetes Mellitus. North American Journal of Medical Sciences [Internet]. 2014 [cited 2018 Dec 15]; 6(2):96-101. DOI: https://doi.org/10.4103/1947-2714.127752

9. Correr CJ, Pontarolo R, Melchiors AC, Rossignoli P, Fernández-Limós F, Radominski RB. Tradução para o português e validação do instrumento Diabetes Quality of Life Measure (DQOL-Brasil). Arq Bras Endocrinol Metab [Internet]. 2008 [cited 2018 Dec 15]; 52(3):515-22. DOI: http://dx.doi.org/10.1590/S0004-27302008000300012

10. Silva REG, Morais ACS, Godoi CD, Garcia OAG, Neto WNR. Avaliação sensório-motora e sua correlação com qualidade de vida em portadores de diabetes mellitus. South American Journal of basic Education, technical and technological [Internet]. 2017 [cited 2018 Dec 15]; 4(1):118-32. Available from: http://revistas.ufac.br/revista/index.php/SAJEBTT/article/view/1140

11. Cunha LL, Mayrink WC. Influência da dor crônica na qualidade de vida em idosos. Rev. dor [Internet]. 2011 [cited 2018 Dec 15]; 12(2):120-4. DOI: http://dx.doi.org/10.1590/S1806-00132011000200008

12. Lima, L.R., Stival, M.M., Funghetto, S.S. Volpe, C.R.G,V., Rehem, T. C. M. S. B., Santos, W. S., et al. Lower quality of life, lower limb pain with neuropathic characteristics, female sex, and ineffective metabolic control are predictors of depressive symptoms in patients with type 2 diabetes mellitus treated in primary care. Int J Diabetes Dev Ctries [Internet]. 2018 [cited 2018 Dec 15]; 8(38):1-8. DOI: https://doi.org/10.1007/s13410-018-0667-5

13. Silva JA, Ribeiro-Filho NP, Matsuhima EH. Mensurando o quinto sinal vital: a dor. Ribeirão Preto: FUNPEC-Editora; 2010. 144p.

14. Fleck MPA, Louzada S, Xavier M, Chachamovich E, Vieira G, Santos L et al. Aplicação da versão em português do instrumento abreviado de avaliação da qualidade de vida "WHOQOL-bref". Rev. Saúde Pública [Internet]. 2000 [cited 2018 Dec 15]; 34(2):178-83. DOI: http://dx.doi.org/10.1590/S0034-89102000000200012

15. Fleck MPA, Chachamovich E, Trentini C. Development and validation of the Portuguese version of the WHOQOL-OLD module. Rev. Saúde Pública [Internet]. 2006 [cited 2018 Dec 15]; 40(5):785-91. DOI: http://dx.doi.org/10.1590/S003489102006000600007 
16. Aguiar FLXS, Ramos LFP, Bichara CNC. Detecção de dor com características neuropáticas em pacientes com diabetes mellitus atendidos na atenção básica. BrJP [Internet]. 2018 [cited 2018 Dec 15]; 1(1):15-20. DOI: http://dx.doi.org/10.5935/25950118.20180005

17. Araújo KO, Andrade AN, Costa TS, Freitas MA, Nascimento MMP, Silva EN. Avaliação da qualidade de vida de portadores de diabetes mellitus tipo 2. Rev enferm UFPE on line [Internet]. 2013 [cited 2018 Dec 15]; 7(9):5583-9. DOI: http://dx.doi.org/10.5205/reuol.3529-29105-1-SM.0709201328

18. Alves ECS, Souza LPS, Alves WS, Oliveira MKS, Yoshitome AY, Gamba MA. Condições de saúde e funcionalidade de idosos com Diabetes Mellitus tipo 2 na Atenção Primária à Saúde. Enfermería Global [Internet]. 2014 [cited 2018 Dec 15]; 13(2):19-36. Available from: http://revistas.um.es/eglobal/article/viewFile/167011/160151

19. Matias COF, Matias COF, Alencar BR. Qualidade de vida em idosos portadores de Diabetes Mellitus Tipo 2 atendidos em Unidades Básicas de Saúde de Montes Claros/MG. Rev Bras Qual Vida [Internet]. 2016 [cited 2018 Dec 15]; 8(2):119-29. DOI: https://doi.org/10.3895/rbqv.v8n2.3841

20. Bouhassira D, Letanoux $M$, Hartemann A. Chronic pain with neuropathic characteristics in diabetic patients: A French crosssectional study. PLOS One [Internet]. 2013 [cited 2018 Dec 15]; 8(9):1-9. DOI: https://doi.org/10.1371/journal.pone.0074195

21. Geelen CC, Kindermans HP, Bergh JP, Verbunt JA, Perceived Physical Activity Decline as a Mediator in the Relationship Between Pain Catastrophizing, Disability, and Quality of Life in Patients with Painful Diabetic Neuropathy. Pain Practice [Internet]. 2017 [cited 2018 Dec 15]; 17(3):320-8. DOI: https://doi.org/10.1111/papr.12449

22. Sousa MC, Dias FA, Nascimento JS, Tavares DMS. Correlation of quality of life with the knowledge and attitude of diabetic elderly. Invest Educ Enferm [Internet]. 2016 [cited 2017 feb]; 34(1):180-8. doi: http://dx.doi.org/10.17533/udea.iee.v34n1a20

23. Paiva MHP, Pegorari MS, Nascimento JS, Santos AS. Fatores associados à qualidade de vida de idosos comunitários da macrorregião do Triângulo do Sul, Minas Gerais, Brasil. Cien. saúde coletiva [Internet]. 2015 [cited 2018 Dec 15]; 21(11):334756. DOI: http://dx.doi.org/10.1590/1413-812320152111.14822015

24. Ferretti F, Castanha AC, Padoan ER, Lutinski J, Silva MR. Qualidade de vida de idosos com e sem dor crônica. BrJP [Internet] 2018 [cited 2018 Dec 15]; 1(2):111-5. DOI: http://dx.doi.org/10.5935/2595-0118.20180022

25. Diretrizes da Sociedade Brasileira de Diabetes 2017-2018. Organização José Egídio Paulo de Oliveira. São Paulo: Editora Clannad [Internet]. 2017 [cited 2018 Dec 15]. Available from: https://www.diabetes.org.br/profissionais/images/2017/diretrizes/diretrizes-sbd-2017-2018.pdf

26. Sardinha AHL, Verzaro PM, Costa LLN, Murici AFF, Falcão BCS. Avaliação da qualidade de vida de idosas com diabetes mellitus. Rev enferm UFPE on line [Internet]. 2018 [cited 2018 Dec 15]; 12(2):345-56. DOI: https://doi.org/10.5205/1981-8963v12i2a23164p345-356-2018

27. Miranzi SSC, Ferreira FS, Iwamoto HH, Pereira GA, Miranzi MAS. Qualidade de vida de indivíduos com diabetes mellitus e hipertensão acompanhados por uma equipe de saúde da família. Texto contexto-enferm [Internet]. 2008 [cited 2016 Dec 15]; 17(4):672-9. DOI: http://dx.doi.org/10.1590/S0104-07072008000400007

28. Tavares DMS, Santos ÉA dos, Dias FA, Ferreira PCS, Oliveira PB. Factors associated with quality of life of elderly people with diabetes mellitus. J Nurs UFPE on line [Internet]. 2014 [cited 2018 Jan 15]; 8(6):1491-501. DOI: http://dx.doi.org/10.1590/198122562017020.16014

29. Santos CM, Hugo FN, Leal AF, Hilgert JB. Comparison of two assessment instruments of quality of life in older adults. Rev Bras Epidemiol [Internet]. 2013 [cited 2018 Dec 15]; 16(2):328-37. DOI: http://dx.doi.org/10.1590/S1415-790X2013000200009

30. Nogueira MF, Magalhães LD, Maia AKF, Torquato IMB, Trigueiro JS, Alves MSCF. Quality assessment of elderly living with diabetes mellitus. J Nurs UFPE on line [Internet]. 2015 [cited 2018 Dec 15]; 9(6):8688-97. DOI: https://doi.org/10.5205/19818963-v9i6a10646p8688-8697-2015

31. Reis LA, Torres GV, Reis LA, Oliveira LS, Sampaio LS. Avaliação da qualidade de vida em idosos portadores de diabetes mellitus tipo 2. Rev Eletr. FAINOR [Internet]. 2009 [cited 2018 Dec 15]; 2(1):64-76. Available from: http://srv02.fainor.com.br/revista/index.php/memorias/article/view/61/37

32. Ribeiro AP, Souza ER, Atie S, Souza AC, Schilithz AO. The influence of falls on the quality of life of the aged. Ciênc. saúde coletiva [Internet]. 2008 [cited 2018 Dec 15]; 13(4):1265-73. DOI: http://dx.doi.org/10.1590/S1413-81232008000400023

33. Tavares DMS, Côrtes RM, Dias FA. Quality of life of elderly with diabetes mellitus. Cienc Cuid Saude [Internet]. 2011 [cited 2018 Dec 15]; 10(2):290-7. DOI: http://dx.doi.org/10.4025/cienccuidsaude.v10i2.10888

34. Sousa EL, Martins MM, Costa MS, Moreira MRC, Silva AO. Quality of life and factors associated with the health of elderly diabetics. Rev enferm UERJ [Internet]. 2016 [cited 2018 Dec 15]; 24(5):e8456. DOI: http://dx.doi.org/10.12957/reuerj.2016.8456

35. Gambin, G. Qualidade de vida de idosos do meio rural usuários da estratégia de saúde da família. Porto Alegre. Tese [Mestrado em Enfermagem] - Universidade Federal do Rio Grande do Sul; 2013. [cited 2018 Dec 15]. 130p. Available from: https://www.lume.ufrgs.br/bitstream/handle/10183/81788/000906219.pdf?sequence $=1$

36. Kolawole BA, Mosaku SK, Ikem RT. A Comparison of two measures of quality of life of Nigerian clinic patients with type 2 Diabetes Mellitus. African Health Sciences [Internet]. 2009 [cited 2018 Dec 15]; 9(3):161-6. Available from: https://www.ncbi.nlm.nih.gov/pmc/articles/PMC2887035/

37. Franco Júnior AJA, Viana HMG, Pereira AL. Quality of life and glycemic control of patients of type 2 Diabetes Mellitus. Rev. Psicol. Saúde [Internet]. 2013 [cited 2018 Dec 15]; 5(2):102-8. Available from: http://pepsic.bvsalud.org/pdf/rpsaude/v5n2/v5n2a05.pdf

38. Paula GR, Souza BN, Santos LF, Barbosa MA, Brasil VV, Oliveira LMAC. Quality of life assessment for health promotion groups. Rev Bras Enferm [Internet]. 2016 [cited 2018 Dec 15]; 69(2):242-9. DOI: http://dx.doi.org/10.1590/0034-7167.2016690206i 